\title{
Repeated Renal Biopsy - A Predictive Tool to Assess the Probability of Renal Flare in Lupus Nephritis
}

\author{
Gastón J. Piñeiro ${ }^{a}$ Pilar Arrizabalaga ${ }^{a}$ Manel Solé ${ }^{b}$ Rosa M. Abellana ${ }^{c}$ \\ Gerard Espinosa $^{d}$ Ricard Cervera ${ }^{d}$ \\ Departments of a Nephrology and Renal Transplant, ${ }^{b}$ Pathology, ${ }^{c}$ Public Health, and ${ }^{\mathrm{d}}$ Autoimmune Diseases, \\ Hospital Clinic, University of Barcelona, Institut d'Investigacions Biomèdiques August Pi i Sunyer (IDIBAPS), \\ Barcelona, Spain
}

\section{Key Words}

Lupus nephritis · Repeated renal biopsy .

True or apparent remission

\begin{abstract}
Background: How one responds to treatment of lupus nephritis (LN) is based on clinical features, but the activity in renal biopsy (RB) is uncertain. We have described the therapeutic decisions after performing a repeated $\mathrm{RB}$ on the assessment of response to intravenous cyclophosphamide (IC) and the possible prognostic role of this repeated RB. Methods: Clinical, laboratory and histological features at the initial RB and repeated RB were analyzed in 35 patients. Results: Data in the initial versus the repeated RB were serum creatinine $1.23 \pm 1.08$ and $0.96 \pm 0.45 \mathrm{mg} / \mathrm{dl}(p<0.05)$, glomerular filtration rate $<60 \mathrm{ml} / \mathrm{min}$ in 12 and $5 \%$ patients and proteinuria $4.1 \pm 2.8$ vs. $0.61 .1 \mathrm{~g} /$ day $(p<0.05)$. Significant differences were detected in hematuria, nephrotic syndrome and serological immune features. Complete renal remission was reached in $60 \%(n=21)$ at the time of the repeated RB, partial remission in $31.4 \%(n=11)$, and no response IC in $8.6 \%(n=3)$. Nine patients showed proliferative forms in the repeated RB, 3 of them had proteinuria $<1 \mathrm{~g} /$ day. Just after the repeated RB, $34.3 \%$ increased or
\end{abstract}

started a new immunosuppressive therapy, $17.1 \%$ remained with the same complementary IST, and $14.3 \%$ decreased or stopped it. In the follow-up post repeated RB, $34.5 \%$ without active lesions showed a renal flare versus $77.8 \%$ with active lesions ( $p=0.04)$. The mean time was 120 and 45 months, respectively. Conclusion: A repeated biopsy in LN distinguishes patients in true remission from those in apparent remission. By doing this, we can identify patients who could benefit from intensified treatment and for whom unnecessary treatment methods can be modified or eliminated.

(c) 2016 S. Karger AG, Basel

\section{Introduction}

The incidence of lupus nephritis (LN) was $16 \%$ at the time of diagnosis of systemic lupus erythematosus (SLE) and $39 \%$ throughout the evolution [1], with a negative impact on survival, but in more than $80 \%$ of these patients, LN was present at the time of enrolment [2]. Although the mortality of SLE has improved, renal survival patterns in LN over the past decades remain unchanged [3].

There is agreement that renal biopsy $(\mathrm{RB})$ is essential to establish the diagnosis and propose treatment methods

\section{KARGER}

E-Mail karger@karger.com

www.karger.com/ajn
(C) 2016 S. Karger AG, Basel

0250-8095/16/0446-0439\$39.50/0
Pilar Arrizabalaga, MD, PhD

Department of Nephrology and Renal Transplant Hospital Clínic

Villarroel 170, ES-08036 Barcelona, Catalonia (Spain)

E-Mail parriza@ clinic.ub.es 
[4]. However, the assessment of response to treatment is based on clinical and laboratory features, and the definitions of response are varied in several guidelines [5-7]. New data pointed out that patients with LN may have inflammatory activity in the renal tissue without clinical signs of renal involvement or despite apparent good clinical response to therapy $[8,9]$.

Repeated RB after immunosuppressive treatment may be important to distinguish patients in histopathological remission (true remission) of those in apparent remission, identifying patients who may need intensified therapy. Despite this data, there is no consensus about the need to repeat RB to assess the response to immunosuppressive treatment $[5-7,10]$.

The objective of this study was to describe the therapeutic decisions after a repeated $\mathrm{RB}$, taking into account the clinical and histopathological changes after treatment with intravenous cyclophosphamide (IC). This analysis also aimed at assessing the possible prognostic role of this repeated $R B$.

\section{Subjects and Methods}

\section{Patients}

We retrospectively reviewed the database of Departments of Autoimmune Disease and Nephrology of Hospital Clinic, Barcelona, from 1990 to 2009 to identify patients with LN class III or IV that were treated with an induction regimen of IC and in those repeated RB was performed. All the patients fulfilled at least 4 of the American College of Rheumatology (ACR) criteria for the diagnosis of SLE at the time of kidney biopsy. Patients without histological confirmation of LN or without induction therapy with IC were excluded. The decision to perform a repeated biopsy and the time that it had to be performed were based on clinical judgment and not on an established protocol.

The Barcelona Clinic hospital's Ethics Committee approved the study protocol under the number 2016/0133.

\section{Clinical and Laboratory Variables}

The following clinical and serological data from each patient were collected from the medical records: age, gender, duration of SLE (time from the diagnosis of SLE to initial RB), time from the first IC pulse to repeated RB, hypertension (systolic blood pressure $\geq 140 \mathrm{~mm} \mathrm{Hg}$ and/or diastolic blood pressure $\geq 90 \mathrm{~mm} \mathrm{Hg}$ ), albumin and cholesterol serum levels, anti-double-stranded (ds) DNA antibody, C3, C4 and CH50, lupus anticoagulant, IgG anticardiolipin (aCL) antibodies and IgM aCL antibodies.

Renal evaluation included urinary sediment and investigation of $24 \mathrm{~h}$ urine protein excretion. Hematuria (presence of $\geq 5$ red blood cells per field $(100 \times)$ in an isolated urine sample at least in 2 determinations), proteinuria (presence of $\geq 0.5 \mathrm{~g} / \mathrm{dl}$ of protein in $24 \mathrm{~h}$ at least in 2 determinations), and nephrotic syndrome (urinary protein level $>3.5 \mathrm{~g} / 24 \mathrm{~h}$, albumin serum $<30 \mathrm{mg} / \mathrm{dl}$ and low-density lipoprotein cholesterol $>200 \mathrm{mg} / \mathrm{dl}$ ) were all assessed.
Renal function was determined by the serum creatinine ( $\mathrm{mg} / \mathrm{dl}$ ) level and by the estimated glomerular filtration rate (eGFR) using the Modification of Diet in Renal Disease (MDRD) formula [11]. Chronic kidney disease was defined as a condition when eGFR $<60$ $\mathrm{ml} / \mathrm{min}[12]$.

Clinical and laboratory features at the time of initial RB and repeated $\mathrm{RB}$ were analyzed and compared.

\section{Treatments}

Treatment methods with intravenous pulses of cyclophosphamide, steroids, another immunosuppressive treatment, anti-malarial drugs, and inhibitors of the renin-angiotensin system were recorded.

\section{Definition of Renal Response and Relapse}

Response was defined according to the Kidney Disease: Improving Global Outcomes (KDIGO)-Practice Guideline [7] and the Consensus document from the Systemic Autoimmune Disease Group of the Spanish Society of Internal Medicine and the Spanish Society of Nephrology [10] on the terminology used in the management of LN. A complete response (CR) is defined as nonactive urinary sediment with proteinuria $\leq 0.5 \mathrm{~g}$ /day and normal or stable renal function (whether GFR was normal or not, a greater variation than $15 \%$ of normal of the filtrate was previously altered). Partial response was defined in patients with baseline proteinuria $<3.5 \mathrm{~g} /$ day, reduction of proteinuria in $>50 \%$ in comparison with the initial, and as decrease in proteinuria $<3.5 \mathrm{~g} /$ day in patients with nephrotic syndrome. In both situations, inactive sediment and stabilization or improvement $( \pm 25 \%)$ in GFR with respect to initial values were needed.

We conclude that there is an 'established kidney damage' when there is the presence of an eGFR $\leq 50 \mathrm{ml} / \mathrm{min}$ and/or proteinuria $\geq 3.5 \mathrm{~g} / 24 \mathrm{~h}$ maintained for 6 months and/or end-stage renal disease (ESRD) with or without replacement therapy as established in Systemic Lupus International Collaborative Clinics Damage Index [13].

For each patient, the duration of follow-up was considered to be the time from repeated RB to the last medical visit. In addition, the development of renal failure, ESRD requiring dialysis or renal transplantation, and death were also recorded.

Relapse or renal flare was defined based on the suggestions of the guide KDIGO [7] and the Spanish consensus [10], by the presence of glomerular hematuria $(<5$ to $>15$ red blood cells per field), deterioration on renal function (if baseline creatinine is: $<2$ $\mathrm{mg} / \mathrm{dl}$, an increase of $0.2 \mathrm{mg} / \mathrm{dl}$; or if $>2 \mathrm{mg} / \mathrm{dl}$, an increase of 0.41 $\mathrm{mg} / \mathrm{dl}$ ), or increase in urinary albumin/creatinine ratio (if baseline $<500 \mathrm{mg} / \mathrm{g}$, an increase to $\geq 1,000 \mathrm{mg} / \mathrm{g}$, if baseline $500-1,000 \mathrm{mg} / \mathrm{g}$, an increase to $\geq 2,000 \mathrm{mg} / \mathrm{g}$, if baseline $>1,000 \mathrm{mg} / \mathrm{g}$, an increase of $\geq$ twofold).

\section{Histological Features}

Retrospective evaluation of RB was performed using the original slides processed for routine conventional light microscopy. Specimens were fixed in Bouin or buffered formalin and embedded in paraffin. Sections were stained with hematoxylin-eosin, periodic acid-Schiff, Jones' methenamine silver and Masson's trichrome. Immunofluorescence results were retrieved from the original reports.

Each biopsy sample was retrospectively evaluated by a pathologist who was blinded to the status and management of the patient; 
Table 1. Demographic features of the 35 patients with LN

\begin{tabular}{lc}
\hline & $\mathrm{n}=35$ \\
\hline Age, years & $31.7 \pm 12.3$ \\
Gender, female & $32(91)$ \\
Time from SLE diagnosis to initial RB, months & $42.6 \pm 52.8$ \\
Time from first intravenous cyclophosphamide pulse to repeated RB, months & $30.5 \pm 9.3$ \\
Follow up from repeated RB, years & $9.2 \pm 6.4$ \\
\hline
\end{tabular}

Values of categorical variables are the number and percentage of patients for whom continuous variables are presented as mean $\pm \mathrm{SD}$.

evaluation was done according to the World Health Organization (WHO) classification of LN when the biopsy was performed before the year 2003, and according to the International Society of $\mathrm{Ne}$ phrology/Renal Pathology Society (ISN/RPS) classification of LN after that date. If biopsy specimens were classified according to the WHO classification, they were reassessed according to the ISN/RPS classifications, and the new classification was compared between successive biopsies. Specimens were evaluated to understand the degree of interstitial cellular infiltration, tubular atrophy, interstitial fibrosis, and nephroangioesclerosis. The interstitial cellular infiltration was assessed as negative (-) when no cells were seen; positive $(+)$ when cellular infiltration was mild; ++ when cellular infiltration was moderate; +++ when cellular infiltration was severe. The tubular atrophy, interstitial fibrosis and nephroangioesclerosis were assessed as negative (-) when absent; + when less than $25 \%$ of the tubuli, interstitium or vessels were affected; ++ when the lesion was between 25 and 50\%, and +++ when the lesion affected more than $75 \%$ of tubuli, interstitium or vessels. Activity (0-24) and chronicity (0-12) indices from each biopsy sample were assigned [14].

Antiphospholipid antibodies-associated nephropathy consists of whole renal vascular involvement. Histological findings are thrombotic microangiopathy, which basically represents an acute event. Other lesions constitute fibrous intimal hyperplasia, fibrocellular arterial occlusion, focal cortical atrophy and thyroidization tubular reflect chronic renal damage [15].

\section{Statistical Analysis}

Quantitative variables were described using mean and SD, except the renal flare-free after repeated RB that is expressed using median, and qualitative variables were described in terms of number and percentage of patients. We used the Student's test paired test and McNemar's test to compare variables at baseline and follow-up; and for variables with non-normal distribution, we used Wilcoxon ranksum test, a non-parametric test. The laboratory results and renal response are compared based on the presence of proliferative lesions (PL) in the repeated RB using the Student's test, chi-square, and Fisher's exact test in case number of expected accounts was lower than 5 .

The Kaplan-Meier estimator was used to estimate the probability distribution of not developing a renal flare after repeated RB in patients based on the existence of $\mathrm{PL}$, and the comparison between groups was performed using the log rank test.

Statistical significance was set at the level of 0.05 . Statistical analysis was performed using IBM SPSS Statistics version 19 (SPSS Inc., Armonk, N.Y., USA).

\section{Results}

\section{Patients}

Analysis was performed for 35 patients with proliferative LN; it was performed for those who received IC as induction therapy and for those where a repeated $\mathrm{RB}$ was performed (table 1). Table 2 summarizes the main clinical and laboratory features at the time of initial and repeated RB.

\section{Treatment of LN}

Sixteen (46\%) patients received National Institutes of Health IC regimen [16], 7 (20\%) received low-dose Euro-Lupus IC regimen [17], mean cumulative dose of $7.7 \pm 3.6 \mathrm{~g}$ (range 3-13.6 g). Twelve (34.3\%) patients received IC variables regimens with similar dose accumulated $8.3 \pm 2.7 \mathrm{~g}$ (range $3-12.5 \mathrm{~g}$ ). The overall mean $\mathrm{cu}-$ mulative dose of IC was $7.9 \pm 3.3 \mathrm{~g}$. Also, the number of IC doses was similar in $\mathrm{NIH}+$ Euro-Lupus regimens mean 8.8 (range 6-12) and in the variable IC regimens mean 8.9 (range 6-12).

Complications related to IC were reported in 22 (63\%) patients; 10 of them (29\%) presented urinary tract infections, 8 (23\%) amenorrhea, 7 (20\%) leukopenia, 4 (11\%) herpes zoster, 1 (3\%) toxic hepatitis, and 1 (3\%) pancytopenia. However, these complications were not associated with the accumulated IC dose $(p=0.21)$ or age of the patients $(\mathrm{p}=0.51)$.

Regarding corticosteroids all patients received oral prednisone, with an initial mean dose of $56 \mathrm{mg} /$ day (range 50-70 mg/day), tapering according to clinical response until $10.25 \pm 13 \mathrm{mg} /$ day as dose of maintenance at the time of repeated $\mathrm{RB}$. The cumulative dose of prednisone between the initial $R B$ and the repeated $R B$ was $11 \pm 4 \mathrm{~g} /$ day/ number of days. At the start of the treatment, intravenous high-doses of methylprednisolone $(2.8 \pm 0.4 \mathrm{~g})$ were used in $16(46 \%)$ patients due to cellular crescents. In addition, 
Table 2. Clinical and laboratory features of the 35 patients with $\mathrm{LN}$ at the time of initial and repeated RB

\begin{tabular}{lccr}
\hline & Initial RB & Repeated RB & p value \\
\hline Arterial hypertension & $21(60)$ & $19(54)$ & $\mathrm{ns}$ \\
Serum creatinine, $\mathrm{mg} / \mathrm{dl}$ & $1.23 \pm 1.08$ & $0.96 \pm 0.45$ & 0.034 \\
MDRD $<60 \mathrm{ml} / \mathrm{min} / 1.73 \mathrm{~m}^{2}$ & $12(34.3)$ & $5(14.3)$ & 0.092 \\
Proteinuria, $\mathrm{mg} / 24 \mathrm{~h}$ & $4,114.74 \pm 2,896.84$ & $623.8 \pm 1,136.85$ & $<0.005$ \\
Haematuria & $28(80)$ & $7(20)$ & $<0.005$ \\
Nephrotic syndrome & $20(57.1)$ & $1(2.9)$ & $<0.005$ \\
Anti-dsDNA antibodies, UI/ml & $165.05 \pm 60.71$ & $57.74 \pm 56.52$ & $<0.005$ \\
CH50, U/ml & $22.03 \pm 15.1$ & $46.29 \pm 15.72$ & $<0.005$ \\
Low C3 & $34(97.1)$ & $11(31.2)$ & $<0.005$ \\
Low C4 & $29(82.9)$ & $8(22.9)$ & $<0.005$ \\
Antiphospholipid antibodies & $16(45.7)$ & $8(22.9)$ & 0.021 \\
\hline
\end{tabular}

Values of categorical variables are the number and percentage of patients for whom continuous variables are presented as mean $\pm \mathrm{SD}$.

$\mathrm{ns}=$ Not significant.

$27(77 \%)$ patients received antimalarial drugs at the time of initial RB.

Twenty-one patients received only IC as immunosuppressive therapy. After IC, 14 (40\%) patients were under the immunosuppressive maintenance treatment; of them 6 were treated with azathioprine, 6 with MMF, and 2 patients with both. The mean number of IC pulses was 9 (range 6-12).

\section{Histological Features}

Table 3 describes the histological characteristics in the initial RB and in the repeated RB. At least 10 glomeruli were available in $82 \%$ of patients in the initial $\mathrm{RB}$ and in the $46 \%$ of patients in the repeated RB. Of note, $26(74.3 \%)$ patients presented non-PL (NPL) in the repeated RB, whereas $9(25.7 \%)$ did it. Five of them (14.3\%) showed ISN class III A/C and 4 (11.4\%) patients showed ISN class IV A/C.

Laboratory findings at the time of repeated $\mathrm{RB}$ are collected in table 4 based on the presence of PL or NPL. Patients with PL presented with higher levels of $24 \mathrm{~h}$ proteinuria $(1,667 \pm 1,919$ vs. $262 \pm 229 \mathrm{mg} ; \mathrm{p}=0.001)$ and lower levels of C4 $(0.14 \pm 0.09$ vs. $0.30 \pm 0.17 \mathrm{~g} / \mathrm{l} ; \mathrm{p}=$ 0.003). Furthermore, all patients with NPL were clinical responders; 20 (77\%) of them achieved a CR and $6(23 \%)$ a partial response. One out of $9(11 \%)$ patients with PL was in complete remission, $5(55 \%)$ patients were in partial remission and $3(33 \%)$ patients sustained no response. There were no differences in the induction treatment between the group with proliferative and NPL, and the time interval between initial treatment and second $\mathrm{RB}$ was similar in both groups $(p=0.7)$. In PL, the mean time was 30.5 months (range 13-56), and in NPL, it was 27.4 months (range 13-40).

\section{Therapeutic Decisions after Repeated $R B$}

At the time of repeated RB, 21 (60\%) patients developed CR, $11(31 \%)$ patients developed partial response and $3(9 \%)$ patients were non-responders. All patients who developed CR showed NPL, but 5 out of 11 patients who developed partial response showed PL in the repeated RB.

As far as treatment modalities are concerned, all patients were undergoing treatment with prednisone at the time of the repeated RB. Twelve patients (34\%) who received oral prednisone alone of maintenance continued the same. Just after the repeated RB, 6 (17\%) patients remained with the same complementary immunosuppressive therapy, 5 (14\%) decreased its frequency or stopped it, and 12 (34\%) increased or started a new IST; in 3 out of these patients the indication was because of extra renal SLE activity.

\section{Long-Term Follow-Up Based on the Results of Repeated $R B$}

Seven out of 9 (78\%) patients with PL and 9 out of 26 (34.5\%) patients with NPL in the repeated RB developed renal flare throughout the follow-up. Of note, the probability of renal flare throughout the first 2 years after the repeated RB was similar in both groups. However, after 48 months, the probability of renal flare-free was much lower in the group with PL than in the group with NPL, 0.375 vs. 0.799 , respectively (fig. 1 ). 
Table 3. Histological features of the study population at the time of the initial and repeated RB

\begin{tabular}{|c|c|c|c|}
\hline & Initial RB & Repeated RB & $\mathrm{p}$ value \\
\hline Number of glomeruli & $13 \pm 6.35$ & $9.7 \pm 4.65$ & 0.027 \\
\hline ISN class: III and IV; A or A/C & $33(94.3)$ & $8(22.9)$ & $<0.005$ \\
\hline ISN $3 \mathrm{~A} / \mathrm{C}+\mathrm{V}$ & & $1(2.9)$ & \\
\hline ISN IV A + V & $2(5.7)$ & & \\
\hline No LN & & $2(5.7)$ & \\
\hline ISN class: I & & $1(2.9)$ & \\
\hline ISN class: II & & $11(31.4)$ & \\
\hline ISN IIIC & & $8(22.9)$ & \\
\hline ISN IVC & & $3(8.6)$ & \\
\hline ISN VI & & $1(2.9)$ & \\
\hline Activity index & $9.94 \pm 3.39$ & $1.32 \pm 1.96$ & $<0.005$ \\
\hline Chronicity index & $1.57 \pm 1.61$ & $2.47 \pm 1.79$ & 0.001 \\
\hline NGEP/NG & $0.18 \pm 0.185$ & $0.032 \pm 0.019$ & $<0.005$ \\
\hline NGFEP/NG & $0.032 \pm 0.09$ & $0.045 \pm 0.13$ & 0.94 \\
\hline NGS $>80 \% / \mathrm{NG}$ & $0.04 \pm 0.086$ & $0.18 \pm 0.15$ & $<0.005$ \\
\hline Interstitial leukocyte infiltration $(1+2)^{*}$ & $17(48.6)$ & $6(17.6)$ & 0.001 \\
\hline Interstitial fibrosis $(1+2)^{* *}$ & $22(62.9)$ & $22(62.9)$ & 1 \\
\hline Tubular atrophy $(1+2)^{* *}$ & $17(48.6)$ & $17(48.6)$ & 1 \\
\hline Nephroangioesclerosis $(1)^{* *}$ & $5(14.3)$ & $5(14.3)$ & 1 \\
\hline Thrombotic microangiopathy & 0 & $2(5.7)$ & $>0.05$ \\
\hline Chronic injury of aPLN & $1(2.9)$ & $1(2.9)$ & 1 \\
\hline
\end{tabular}

Values of categorical variables are the number and percentage of patients for whom continuous variables are presented as mean \pm SD.

* Interstitial cellular infiltration assessed as $1+$ : mild and $2++$ : moderate.

** Tubular atrophy, interstitial fibrosis and nephroangioesclerosis assessed as $1+$ : less than $25 \%$ and $2++$ : lesion between 25 and $50 \%$ of tubuli, interstitium or vessels.

$\mathrm{A}=$ Active lesions or $\mathrm{A} / \mathrm{C}=$ active/chronic lesions in class III and IV LN according to the ISN classification; NGEP/ $\mathrm{NG}=$ number of glomeruli with extracapillary proliferation/number of glomeruli; NGFEP/NG = number of glomeruli with fibrous extracapillary proliferation/number of glomeruli; NGS $>80 \% / \mathrm{NG}=$ number of glomeuli with glomerulosclerosis $>80 \%$ /number of glomeruli; aPLN $=$ antiphospholipid antibodies associated nephropathy.

Table 4. Laboratory and renal response according the presence of proliferative lesions and chronicity index in the repeated $\mathrm{RB}$

\begin{tabular}{lccc}
\hline & NPL $(\mathrm{n}=26)$ & PL $(\mathrm{n}=9)$ & p value \\
\hline Creatinine, mg/dl & $0.85 \pm 0.232$ & $1.29 \pm 0.74$ & 0.1 \\
Proteinuria, g/24 h & $262.7 \pm 229$ & $1,667 \pm 1,918$ & 0.001 \\
C3, g/l & $1.07 \pm 0.33$ & $0.78 \pm 0.38$ & 0.067 \\
C4, g/l & $0.3 \pm 0.17$ & $0.14 \pm 0.09$ & 0.003 \\
Anti-dsDNA antibodies, UI/ml & $41.76 \pm 36.8$ & $104.11 \pm 77.93$ & 0.056 \\
CR & $20(77)$ & $1(11)$ & 0.0009 \\
Partial response & $6(23)$ & $5(55)$ & 0.1 \\
Non response & 0 & $3(33)$ & 0.05 \\
Chronicity index & $3 \pm 2.44$ & $2.19 \pm 1.55$ & 0.316 \\
\hline
\end{tabular}

Values of categorical variables are the number and percentage of patients for whom continuous variables are presented as mean \pm SD. 


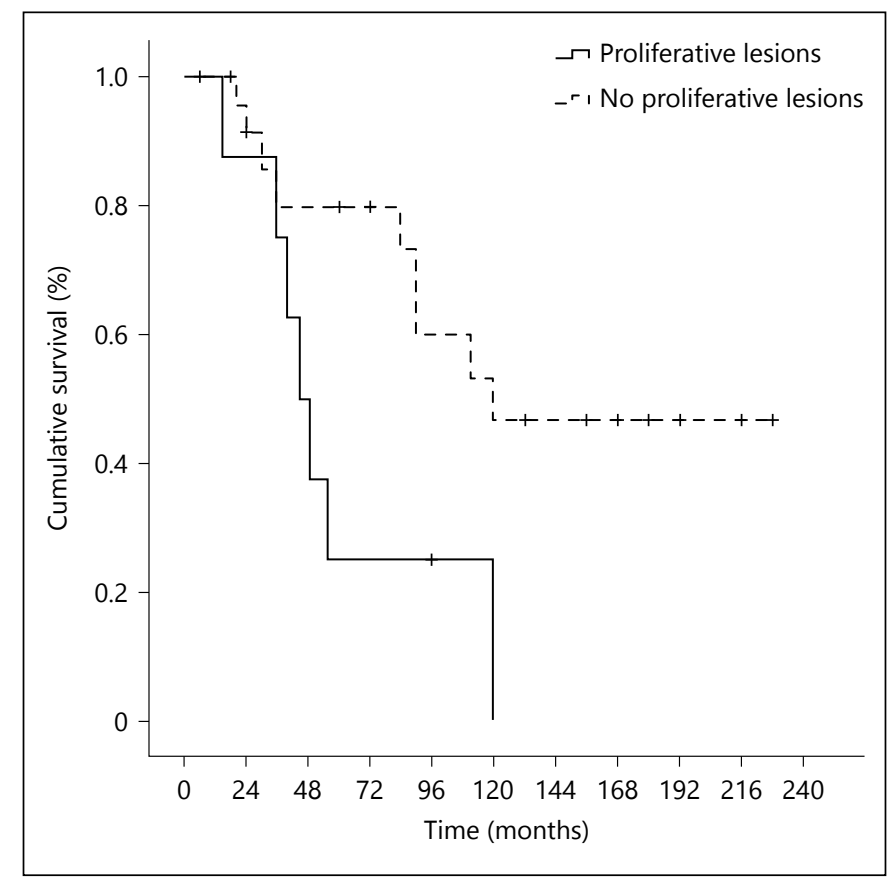

Fig. 1. Probability of not presenting a renal flare after the repeated RB.

In other words, the median time risk over the time of renal flare after repeated $\mathrm{RB}$ was 45 months in patients with PL compared with 120 months in those without PL $(\mathrm{p}=0.046)$.

At the end of follow-up, 7 out of 9 (78\%) patients with PL and 4 out of 26 (15\%) patients with NPL developed glomerular filtration, calculated by MDRD, $<60 \mathrm{ml} /$ $\min / 1.73 \mathrm{~m}^{2}(\mathrm{p}=0.0013)$. In our series, the degree of chronic lesions (glomerulosclerosis, interstitial fibrosis, tubular atrophy) in the repeated RB is not significantly associated with the deterioration of renal function at end of follow-up. Finally, 2 patients in the PL group died due to heart failure in one case and pulmonary bleeding in another case.

\section{Adverse Events Related to RB Performance}

Only $1(3 \%)$ patient presented a severe adverse event in the form of hematoma in the lower pole, self-limited with stable hemoglobin related to the repeated RB.

\section{Discussion}

This is a retrospective study on repeated $\mathrm{RB}$ in $35 \mathrm{pa}-$ tients after induction therapy with IC. Unlike other studies that included patients with NPL and the induction treatment varied, a strong point in this study is that all patients initially presented PL (III-IV A or A/C) and all received similarly induction treatment with IC. A limitation of our study is that the decision and the time to perform a repeated biopsy were based on clinical judgment and not on an established protocol. In general, the decision to perform a second biopsy was to evaluate the histological status prior to a possible therapeutic change. But the time interval between initial treatment and second $\mathrm{RB}$ was no different in the PL and NPL groups ( $\mathrm{p}=0.7)$.

Proteinuria is a common marker of LN activity, and the early reductions in proteinuria have been shown to be important for long-term renal outcomes in LN. The longterm follow-up of the Euro-Lupus Nephritis trial [18] and MAINTAIN trial [19] also notes that reduction in proteinuria is the most significant predictor of good response. After 12 months of treatment, the achievement of proteinuria $<0.7 \mathrm{~g} /$ day best predicted good renal outcome, while the sensitivity and specificity were 71 and $75 \%$, respectively [20]. In spite of this, chronic established lesions or change in ISN class V of LN can explain the reason for persisting elevated proteinuria.

In accordance with these data, higher proteinuria in the PL group versus the NPL group $(\mathrm{p}=0.001)$ were detected at the moment of repeated RB. Interestingly, 3 of 9 patients with PL had proteinuria less than $300 \mathrm{mg} /$ day.

The response criteria in the ACR [4] KDIGO [7] guidelines and European consensus [21] are defined by the decrease in urinary protein, normal or stable renal function and inactive urinary sediment. However, the discrepancy between biopsy results and clinical markers was noted and experts agreed that biopsy was the 'gold standard' for assessing renal activity and felt that more accurate biomarkers than proteinuria were needed for routine management. Repeat RB is helpful to distinguish active LN from chronic damage, in which proteinuria and rising serum creatinine may be present. However, in our series, the presence of chronic lesions (glomerulosclerosis, interstitial fibrosis, tubular atrophy) in the repeated RB is not significantly associated with elevated proteinuria.

As shown by other studies $[8,9,22,23]$, we found persistent histopathological renal activity despite apparent clinical response. Furthermore, PL in repeated RB was associated with more frequent and early risk of renal flare.

The definition of response to treatment based on clinical criteria may affect the results of trials of new treatment methods. A large number of uncontrolled studies [24-27] have suggested the efficacy of rituximab in LN, but it did not improve clinical outcomes in one randomized clinical trial. LUNAR trial [28] was a double-blind, 
placebo-controlled phase III study recruiting patients with active PL LN (class III or IV), which aimed at assessing the efficacy and safety of rituximab in patients treated with mycophenolate mofetil and corticosteroids. The results demonstrated non-significant improvement in the rituximab-treated group, based on the proportion of patients achieving a CR, defined by low levels of proteinuria, a normal serum creatinine level, and negative urine sediment. However, $15 \%$ of patients showed partial response in this group. Limiting the approach to clinical response may be a problem in clinical trials and this could lead to study failure. For this reason, it has been suggested that the realization of the repeated $\mathrm{RB}$ should be considered in the study design of future clinical trials [29].

Effective induction therapy is also expected in the reduction of serologic markers of lupus activity. The reported sensitivity and specificity of anti-dsDNA antibodies and low levels of complement appear as 77 and 73\%, respectively [7]. In our patients, the levels of anti-dsDNA were greater in the PL group than in the NPL group in the repeated RB but not statistically significant. Complement levels were also lower in the PL group than in the NPL group, but only low levels of C4 were statistically significant.

New biomarker development for LN is still in an early stage and the majority of studies have focused on identifying biomarkers that reflect disease activity. But if the disease activity is defined by standard methods, such as proteinuria, urine sediment, and serum creatinine, the novel biomarkers can be no better than these measurements [30]. To improve on standard methods, novel biomarkers must be judged against an activity measurement that is superior such as the kidney biopsy [22].

In our study, just after repeated $\mathrm{RB}, 14 \%(\mathrm{n}=5)$ of patients decreased or stopped IST, and $34 \%(\mathrm{n}=12)$ increased or started a new IST, 6 of them showed proteinuria less than $1 \mathrm{~g} /$ day, and in 3 of these patients, the indication was because of extra renal SLE activity. In 20 out of 32 patients, repeated RB was crucial to adjust the treatment. The discordance between histopathological findings and the laboratory variables routinely used to define disease activity, and also the prognostic information obtained from repeat renal biopsies, emphasize that it is important to evaluate clinical response and adjust treatment in LN.

While the RB after diagnosis is a well-established practice in suspected relapse, the use of this practice in CR or partial response situations is a matter of debate. An RB is an invasive procedure with associated morbidity, that can be performed only if all or patients with certain features should be biopsied. The questions of who should undergo biopsy and when still remain and finally histological response criteria have not been established.

A repeated $\mathrm{RB}$ can be a valuable tool, particularly in patients under partial response, to distinguish patients in true remission from those in apparent remission. This helps in identifying patients who could benefit from intensified treatment, avoiding future irreversible damage, and avoiding unnecessary aggressive treatments when irreversible renal damage is found.

Although the small number of patients included and the retrospective nature of this and other studies in the literature $[8,9]$ make it difficult to arrive at definitive conclusions about the usefulness of the RB in monitoring the response in $\mathrm{LN}$, these data insinuate their utility. Future prospective studies are needed.

\section{Key Messages}

$\mathrm{RB}$ after induction in LN class IV and III distinguishes true from apparent remission.

Repeated RB provides additional understanding; adjust treatment plans to avoid irreversible renal damage or unnecessary aggression.

Repeated RB provides predictive usefulness regarding the risk time of renal flare and guide treatment.

\section{Contributors}

P.A. and G.E.: study design, construction of the database with clinical information and interpretation of results. M.S.: assessment of all the initial and repeated renal biopsies. G.J.P. and R.M.A.: statistical analyses, interpretation of results and manuscript writing. All authors made substantial contribution to the following: drafting the article or revising it critically for important intellectual content; final approval of the version published.

\section{Disclosure Statement}

The authors declare no conflicts of interest.

References

1 Cervera R, Khamashta MA, Font J, Sebastiani GD, Gil A, Lavilla P, et al: Systemic lupus erythematosus: clinical and immunologic patterns of disease expression in a cohort of 1,000 patients. Medicine (Baltimore) 1993;72:113-124.

2 Hanly JG, O’Keeffe AG, Su L, Urowitz MB, Romero-Diaz J, Gordon C, et al: The frequency and outcome of lupus nephritis: results from an international inception cohort study. Rheumatology (Oxford) 2016;55:252-262. 
3 Croca SC, Rodrigues T, Isenberg DA: Assessment of a lupus nephritis cohort over a 30year period. Rheumatology (Oxford) 2011;50: 1424-1430.

4 Liang M, Schur P, Fortin P, St Clair E, Balow J, Costenbader K, et al: The American College of Rheumatology Response Criteria for proliferative and membranous renal disease in systemic lupus erythematosus clinical trials. Arthritis Rheum 2006;54:421-432.

5 Hahn BH, McMahon MA, Wilkinson A, Wallace WD, Daikh DI, Fitzgerald JD, et al: American College of Rheumatology guidelines for screening, treatment, and management of lupus nephritis. Arthritis Care Res (Hoboken) 2012;64:797-808.

6 Bertsias GK, Tektonidou M, Amoura Z, Aringer $\mathrm{M}$, Bajema $\mathrm{I}$, Berden $\mathrm{JH}$, et al: Joint European League Against Rheumatism and European Renal Association-European Dialysis and Transplant Association (EULAR/ ERA-EDTA) recommendations for the management of adult and paediatric lupus nephritis. Ann Rheum Dis 2012;71:17711782.

7 KDIGO Clinical Practice Guideline for Glomerulonephritis: Chapter 12: lupus nephritis. Kidney Int Suppl 2012;2:221-232.

8 Wakasugi D, Gono T, Kawaguchi Y, Hara M, Koseki Y, Katsumata Y, et al: Frequency of class III and IV nephritis in systemic lupus erythematosus without clinical renal involvement: an analysis of predictive measures. J Rheumatol 2012;39:79-85.

9 Zickert A, Sundelin B, Svenungsson E, Gunnarsson I: Role of early repeated renal biopsies in lupus nephritis. Lupus Sci Med 2014; $1: \mathrm{e} 000018$.

10 Ruiz Irastorza G, Espinosa G, Frutos MA, Jiménez Alonso J, Praga M, Pallarés L, et al: Diagnosis and treatment of lupus nephritis. Consensus document from the systemic auto-immune disease group (GEAS) of the Spanish Society of Internal Medicine (SEMI) and Spanish Society of Nephrology (S.E.N.). Nefrologia 2012;32(suppl 1):1-35.

11 Levey AS, Bosch JP, Lewis JB, Greene T, Rogers N, Roth D: A more accurate method to estimate glomerular filtration rate from serum creatinine: a new prediction equation. Modification of Diet in Renal Disease Study Group. Ann Intern Med 1999;130: 461-470.
12 Levey AS, Eckardt KU, Tsukamoto Y, Levin A, Coresh J, Rossert J, et al: Definition and classification of chronic kidney disease: a position statement from Kidney Disease: Improving Global Outcomes (KDIGO). Kidney Int 2005;67:2089-2100.

13 Gladman DD, Goldsmith CH, Urowitz MB, Bacon P, Fortin P, Ginzler E, et al: The Systemic Lupus International Collaborating Clinics/American College of Rheumatology (SLICC/ACR) Damage Index for Systemic Lupus Erythematosus International Comparison. J Rheumatol 2000;27:373-376.

14 D'Agati VD, Jennette JC, Silva FG: Systemic lupus erythematosus; in D'Agati VD, Jennette JC, Silva FG (eds): Non-Neoplastic Kidney Diseases. Washington, American Registry of Pathology, 2005, chapter 13, pp 323-352.

15 Miyakis S, Lockshin MD, Atsumi T, Branch DW, Brey RL, Cervera R, et al: International consensus statement on an update of the classification criteria for definite antiphospholipid syndrome (APS). J Thromb Haemost 2006; 4:295-306.

16 Boumpas DT, Austin HA 3rd, Vaughn EM, Klippel JH, Steinberg AD, Yarboro CH, et al: Controlled trial of pulse methylprednisolone versus two regimens of pulse cyclophosphamide in severe lupus nephritis. Lancet 1992; 340:741-745.

17 Houssiau FA, Vasconcelos C, D’Cruz D, Sebastiani GD, de Ramon Garrido E, Danieli MG, et al: Immunosuppressive therapy in lupus nephritis: the Euro-Lupus Nephritis Trial, a randomized trial of low-dose versus highdose intravenous cyclophosphamide. Arthritis Rheum 2002;46:2121-2131.

18 Houssiau FA, Vasconcelos C, D'Cruz D, Sebastiani GD, de Ramon Garrido E, Danieli MG, et al: The 10-year follow-up data of the Euro-Lupus Nephritis trial comparing lowdose and high-dose intravenous cyclophosphamide. Ann Rheum Dis 2010;69:61-64.

19 Tamirou F, D'Cruz D, Sangle S, Remy P, Vasconcelos C, Fiehn C, et al: Long-term followup of the MAINTAIN nephritis trial, comparing azathioprine and mycophenolate mofetil as maintenance therapy of lupus nephritis. Ann Rheum 2016;75:526-531.

20 Tamirou F, Lauwerys BR, Dall'Era M, Mackay M, Rovin B, Cervera R, et al: A proteinuria cutoff level of $0.7 \mathrm{~g} /$ day after 12 months of treatment best predicts long-term renal outcome in lupus nephritis: data from the MAINTAIN Nephritis Trial.Lupus Sci Med 2015;2:e000123.

21 Gordon C, Jayne D, Pusey C, Adu D, Amoura $\mathrm{Z}$, Aringer $\mathrm{M}$, et al: European consensus statement on the terminology used in the management of lupus glomerulonephritis. Lupus 2009; 18:257-263.

22 Christopher-Stine L, Siedner M, Lin J, Haas M, Parekh H, Petri M, et al: Renal biopsy in lupus patients with low levels of proteinuria. J Rheumatol 2007;34:332-335.

23 Pagni F, Galimberti S, Goffredo P, Basciu M, Malachina S, Pilla D, et al: The value of repeat biopsy in the management of lupus nephritis: an international multicentre study in a large cohort of patients. Nephrol Dial Transplant 2013;28:3014-3023.

24 Moroni G, Gallelli B, Banfi G, Leoni A, Messa $P$ : Rituximab monotherapy for remission induction of proliferative lupus nephritis flares: description of 3 cases. J Nephrol 2010;23:357361.

25 Van Vollenhoven RF, Gunnarsson I, WelinHenriksson E, Sundelin B, Osterborg A, Jacobson SH, et al: Biopsy-verified response of severe lupus nephritis to treatment with rituximab (anti-CD20 monoclonal antibody) plus cyclophosphamide after biopsy-documented failure to respond to cyclophosphamide alone. Scand J Rheumatol 2004;33:423427

26 Ezeonyeji AN, Isenberg DA: Early treatment with rituximab in newly diagnosed systemic lupus erythematosus patients: a steroid-sparing regimen. Rheumatology (Oxford) 2012; 51:476-481.

27 Pepper R, Griffith M, Kirwan C, Levy J, Taube $\mathrm{D}$, Pusey C, et al: Rituximab is an effective treatment for lupus nephritis and allows a reduction in maintenance steroids. Nephrol Dial Transplant 2009;24:3717-3723.

28 Rovin BH, Furie R, Latinis K, Looney RJ, Fervenza FC, Sanchez-Guerrero J, et al: Efficacy and safety of rituximab in patients with active proliferative lupus nephritis: the Lupus $\mathrm{Ne}$ phritis Assessment with Rituximab study. Arthritis Rheum 2012;64:1215-1226.

29 Lightstone L: The landscape after LUNAR: rituximab's crater-filled path. Arthritis Rheum 2012;64:962-965.

30 Rovin $\mathrm{BH}$, Zhang X: Biomarkers for lupus nephritis: the quest continues. Clin J Am Soc Nephrol 2009;4:1858-1865. 